psychosocially complex cases, facilitate multiagency (MA) working and ensure safeguarding of vulnerable young people accessing services.

Aim(s)/objectives To describe characteristics of young people accessing the service and compare those warranting MDTM or MA input to those in whom this was not required.

Methods Retrospective review of electronic patient records of new patients accessing a young people's clinic ( $\leq 18$ years) from January to June 2014. Demographics, clinical and psychosocial details, MDTM case note entries or liaison with other agencies including social services, voluntary sector, mental and other health were analysed. Significance calculation: fisher's exact test. Results 159 cases reviewed. Median age 16 years: female 80\%, locally resident $80 \%$, self-referral $77 \%$, white British $22 \%$, black Caribbean 22\%. 67(42\%) required MA/MDTM working. (45\%, $\mathrm{n}=30 \mathrm{had}$ MA referral/liaison). MA/MDTM patients were more likely to have health adviser input: $57 \%$ vs $21 \% \mathrm{p}=$ 0.0001 , report mental health problems: $33 \%$ vs $3 \% \mathrm{p}=0.0001$, have a social worker: $27 \%$ vs $7 \% \mathrm{p}=0.0003$ or if female, not on contraception: $60 \%$ vs $39 \% \mathrm{p}=0.005$. Amongst those requiring MA/MDTM input $12 \%(\mathrm{n}=8)$ had a safeguarding concern and $7 \%(\mathrm{n}=5)$ were identified as at risk of sexual exploitation.

Discussion/conclusion MDTMs effectively enabled discussion of complex patients. MDTM/MA working was common and such cases were more likely to: lack contraception, need health adviser input, have a social worker and mental health problems highlighting an opportunity for closer working with mental health services.

\section{P223 UNDERSTANDING THE GREATER BURDEN OF STIS AMONG BLACK CARIBBEANS IN THE UK: EVIDENCE FROM A SYSTEMATIC REVIEW}

${ }^{1}$ Sonali Wayal*, ${ }^{1}$ Catherine Griffiths, ${ }^{1}$ Catherine Mercer, ${ }^{1}$ Makeda Gerressu, ${ }^{2}$ Gwenda Hughes. 'University College London, London, UK; ${ }^{2}$ Public Health England, London, UK

10.1136/sextrans-2015-052126.267

Background In the UK, Black Caribbeans are disproportionately affected by STIs.

Aim We conducted a systematic review of attitudinal, behavioural and contextual risk factors of this inequality.

Methods Ten electronic databases were searched for studies on risk factors and drivers of STI among UK Black Caribbeans from 1948 to 30/11/2014. Two independent reviewers screened all identified abstracts and extracted data from selected studies using standardised forms.

Results Of 3220 abstracts identified, 165 were included in the review. STI risk among Black Caribbeans is higher compared to other ethnic groups and varies by gender and age. Being single and reporting first intercourse aged $<16,>1$ new sex partner in the past year, concurrency, and assortative sexual mixing were identified as risk factors. STIs were considered of lower priority than HIV/unplanned pregnancy. Barriers to condom use, especially among women with older and regular partners, were reported. Compared to other ethnic groups, Black Caribbeans were more likely to have ever attended a STI clinic and tested for HIV, but Black Caribbean women were more likely to report delays in seeking care and be sexually active whilst symptomatic. Perceived negative attitudes of clinic staff of the same ethnicity towards young women negatively affected care-seeking.

Discussion/conclusion Sexual behavioural risk factors or access to care did not fully explain the disproportionate STIs burden among Black Caribbeans highlighting the need for further evidence on contextual drivers of STIs. STI reduction interventions should be gender-specific, informed by partnership patterns and address attitudes to STIs and sexual health care-seeking.

\section{P224 THE SEXUAL HEALTH OF THE HOMELESS - AN OUTREACH SEXUAL HEALTH SCREENING PROJECT}

${ }^{1}$ Sarah Stockwell*, ${ }^{1}$ Gillian Dean, ${ }^{2}$ Travis Cox, ${ }^{2}$ Marc Tweed, ${ }^{3}$ Jane Poole, ${ }^{4}$ Georgina Hume, ${ }^{4}$ Steven Nicolson, ${ }^{4}$ Laura Hutchinson. ${ }^{1}$ Brighton and Sussex University Hospitals NHS Trust, Brighton, UK; ${ }^{2}$ Terrance Higgins Trust, Brighton, UK; ${ }^{3}$ Oasis Project, Brighton, UK; ${ }^{4}$ Chlamydia Screening Project, Brighton, UK

\subsection{6/sextrans-2015-052126.268}

Background/introduction Homeless people are at increased risk of STIs, and may struggle to attend conventional services. To improve sexual health access and knowledge for this group, THT launched a weekly outreach testing project for asymptomatic clients in June 2014 at the local homeless service. HIV point of care tests (POCT) and self-taken STI screens (SHS) were offered. Hepatitis $\mathrm{B} / \mathrm{C}$ POCTs were introduced more recently.

Aim(s)/objectives To assess the value of the outreach service and describe project outcomes.

Methods User demographics and testing outcomes were collected at each attendance and reviewed at 6 months.

Results From June to December 2014, 129 clients presented. $83 \%$ were white British, 92\% were male. The mean age was 36 (range 19-65 years). 84\% identified as heterosexual, 14\% bisexual and 2\% homosexual. Only 26\% had previously tested for HIV. Of the asymptomatic service users, $45 \%$ had a HIV test (all negative) and $23 \%$ had a self-taken SHS. Two cases were positive; one urethral chlamydia, one rectal gonorrhoea. Eighteen referrals were made to the local $\mathrm{SH}$ clinic for symptomatic screens, blood-borne virus (BBV) testing, vaccination and contraception. Since introducing hepatitis POCTs 2 weeks ago, 4 clients have tested and 2 were positive for hepatitis C.

Discussion/conclusion Prior to project launch, this client group had significant anxiety regarding HIV and BBV. Having the ability to access a full SH screen in familiar surroundings was welcomed. A significant number of infections have been identified demonstrating the importance of the outreach project, and the need for strong links with mainstream services.

\section{P225 REACH OUT AND TEST ME}

${ }^{1}$ Susanna Currie*, ${ }^{2}$ Melissa Pearson, ${ }^{2}$ Holly Eadsforth, ${ }^{1}$ Orla McQuillan. ${ }^{1}$ Manchester Royal Infirmary, Manchester, UK; ${ }^{2}$ University of Manchester, Manchester, UK

\subsection{6/sextrans-2015-052126.269}

Background Saunas have traditionally been where MSM participate in risky sexual activities, contracting high numbers of sexually transmitted infections (STIs) and have been ideal targets for sexual health outreach work. There has however been a recent trend towards private "Chem-Sex "parties arranged through social media. Is sexual health outreach work in the saunas still justified, particularly in these financially pressured times?

Aim Comparison of outreach services in a large urban centre in 2011 and 2013.

Methods Retrospective case-note review of patients who accessed outreach services July-December 2011 and 2013. 
Results In 2011, 98 case notes were reviewed. The rate of infection was $28.2 \%$.

In 2013, 89 case notes were reviewed. The overall rate of infection fell to $14.6 \%$. However, $46 \%$ had never attended our GUM clinic and among these the infection rate was $22 \%$. The comparative rate in MSM attending clinic was $8.7 \%$. Of those new to our services 19\% had never attended any GU service and of these $82 \%$ had never tested for HIV.

Conclusion Our outreach team tested a significant number of patients with a high burden of infection who had never accessed services. However, the team is taken from conventional clinics; due to staff shortages in the clinic, patients are turned away. A balance needs to be found between financial constraints and reducing infection in hard-to-reach populations. Collaboration with voluntary organisations and saunas will be the key to our success. We are currently setting up a Chem-Sex clinic to target evolving at risk populations.

\section{P226 A YEAR OF 'SEX, STEAM AND STIS'}

${ }^{1}$ Frances Beanland*, 'Sarah Schoeman, ${ }^{2}$ Peter Davis, ${ }^{2}$ Pat McCusker, ${ }^{1}$ Tom Doyle. 'Leeds Teaching Hospital Trust, Leeds, UK; ${ }^{2}$ Yorkshire MESMAC, Leeds, UK

\subsection{6/sextrans-2015-052126.270}

Background/introduction This sauna clinic was set up as recent HIV infection amongst MSM in our city is higher than the national average. Following a successful 6 month pilot, the clinic was commissioned for another year.

$\operatorname{Aim}(\mathrm{s}) /$ objectives

- Provide accessible, convenient sexual healthcare/promotion for 'hard-to-reach' individuals.

- Promote regular STI testing amongst this high-risk group.

- Assess measurable outcomes to determine the service's success.

Methods A weekly nurse-led clinic was set up at the sauna. Rectal, pharyngeal and urine testing for chlamydia and gonorrhoea were offered, with HIV, hepatitis $\mathrm{B} / \mathrm{C}$ and syphilis testing and Hepatitis $B$ vaccination. Identified infections were treated at the sauna clinic or our GUM clinic.

Results 231 new/rebook episodes over 57 clinics. 80\% had previously accessed sexual health services but only $63 \%$ had previously undergone extra-genital sampling. HIV testing uptake was 96\%. 16\% had never tested for HIV; 22\% last tested over a year ago. $20 \%$ reported sex with men and women. $18 \%$ had at least one of chlamydia, gonorrhoea, HIV or syphilis identified, compared with 14\% amongst asymptomatic MSM attending our GUM clinic. $80 \%$ of chlamydia and gonorrhoea infections identified were purely extra-genital. 6 new HIV diagnoses were made, 4 of which were recently acquired HIV. HIV prevalence was 3\%. Discussion/conclusion The service has been continually modified to optimise attendance. A new initiative introduced by the sauna management team includes discounted sauna entry for clients attending the sauna clinic. This clinic's success has been due to close partnership and collaboration between NHS, third sector, private sector and local commissioners.

\section{\begin{tabular}{|l|l}
\hline P227 SEXUAL HEALTH IN TRANS* INDIVIDUALS: HIGH RISK \\
\hline
\end{tabular} AND UNDER REPRESENTED}

Ruth Byrne*, Leigh Chislett, Sheel Patel. Chelsea and Westminster Hospital, London, UK

10.1136/sextrans-2015-052126.27
Background/introduction In the UK, the prevalence of sexually transmitted infections (STI) amongst trans* individuals is unknown. International data estimate HIV prevalence to be as high as $20 \%$. Public health data is lacking primarily due to trans* not being recognised as a gender.

Aim(s)/objectives To identify and characterise trans* individuals within our HIV+ cohort.

Methods Trans* individuals attending for HIV care at three urban care centres were identified by their physician and added to a database. A retrospective review of each electronic patient record was undertaken. Demographics, clinical data and documentation of sexual history and risk behaviours were collated.

Results 23 trans* individuals living with HIV were identified. All were trans"female. 10 (43\%) had a detectable HIV viral load. Within the past 6 months $10(43 \%)$ reported condomless anal sex and $6(26 \%)$ had gonorrhoea and/or chlamydia infection. $11(48 \%)$ were regularly using recreational drugs and $6(26 \%)$ engaged in commercial sex work. 9 (39\%) had no documentation of sexual history.

Discussion/conclusion High levels of vulnerability and specific healthcare needs exist amongst trans* individuals. Within this $\mathrm{HIV}+$ cohort particular concerns include risk of onward transmission of HIV, acquisition of new infections and drug misuse. Our clinic runs a dedicated sexual health, HIV and holistic wellbeing service for trans* individuals that is working to address these issues. Patient record systems need updating to recognise trans* individuals, allowing the prevalence of HIV and other STIs in this group to be accurately recorded. We believe trans* individuals are an at risk group whose healthcare needs should be better addressed.

\section{P228 SEXUALLY TRANSMITTED INFECTIONS - A PREDICTIVE FACTOR FOR CHILD SEXUAL EXPLOITATION?}

Richard Kennedy*, Fiona Fargie. The Sandyford Initiative, Glasgow, UK

\subsection{6/sextrans-2015-052126.272}

Background/introduction Sexually active young people can be at risk of child sexual exploitation (CSE). It has been assumed that the presence of a sexually transmitted infection (STI) should be used a marker of increased risk, however no clear evidence exists to support this.

Aim(s)/objectives We aimed to identify if a relationship exists between the detection of STI and other indicators for CSE, by comparing to a matched control group who tested negative for STI.

Methods Utilising our service's electronic patient record, which automatically prompts staff to risk assess, we identified that 1228 patients aged $\leq 15$ yo were seen between $01 / 04 / 2013$ and 31/03/2014, 52 of whom tested positive for STI. Their notes, plus a control group of 105 patients were reviewed for potential identifiers of CSE.

Results We identified no statistically significant association between testing positive for STI and other predictive factors for CSE.

Discussion/conclusion In this small study we found no significant increase in commonly used indicators for CSE in those who tested positive for STI. This highlights the importance of using several identifiers when assessing for CSE and the need for incorporating alternative screening tools such as Spotting The Signs. 